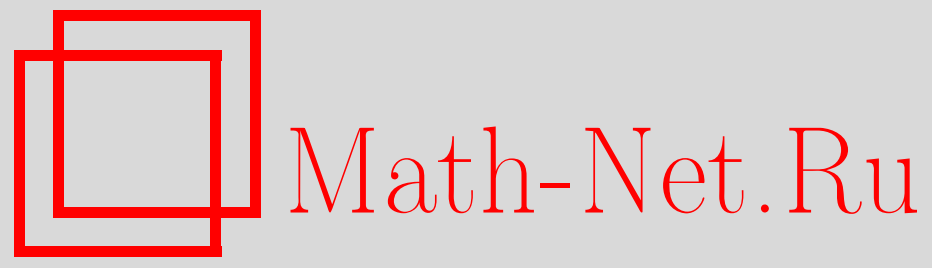

В. Чяканавичюс, Об обобщенных пуассоновских аппроксимациях при моментных ограничениях, Теория вероятн. и ее примен., 1999, том 44, выпуск 1, 74-86

DOI: https://doi.org/10.4213/tvp598

Использование Общероссийского математического портала Math-Net.Ru подразумевает, что вы прочитали и согласны с пользовательским соглашением

http://www . mathnet.ru/rus/agreement

Параметры загрузки:

IP : 3.85 .7 .115

26 апреля 2023 г., 11:42:36

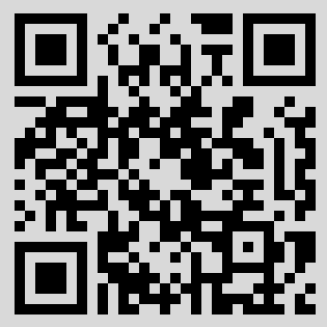




\title{
ОБ ОБОБЩЕННЫХ ПУАССОНОВСКИХ АППРОКСИМАЦИЯХ ПРИ МОМЕНТНЫХ ОГРАНИЧЕНИЯХ
}

\begin{abstract}
Рассматривается аналог первой равномерной теоремы Колмогорова в схеме последовательностей. Частично подтверждена гипотеза Зайцева о точности аппроксимации. В качестве аппроксимаций используются обобщенные распределения Пуассона и обобщенные пуассоновские меры со знаком.
\end{abstract}

Ключевые слова и Фразы: обобщенное распределение Пуассона, вариация, равномерное расстояние.

1. Введение. В настоящей статье рассматривается точность аппроксимации безгранично делимыми законами в равномерной и вариационной метриках. Полученные результаты близки к первой равномерной теореме Колмогорова.

Введем необходимые обозначения. Как обычно, $\mathbf{R}$ и $\mathbf{Z}$ обозначают множества вещественных и целых чисел. Множество всех одномерных распределений обозначим через $\mathscr{F} ; \mathscr{F}_{+}$есть множество распределений, имеющих неотрицательные характеристические функции; D есть множество всех безгранично делимых распределений. Пусть $E_{a}$ означает распределение, сконцентрированное в точке $a, E \equiv E_{0}$. Обозначения $C_{m}$ и $C_{m}(\cdot)$ используются соответственно для положительных абсолютных постоянных и постоянных, зависящих от указанного параметра. Произведения и степени мер понимаются в смысле свертки: $F G=F * G, F^{n}=F^{* n}, F^{0}=E$. Для $F \in \mathscr{F}, h>0$ введем функцию концентрации $Q(F, h)=\sup _{x} F\{[x, x+h]\}$. Для любой меры ограниченной вариации $W$ обозначим через $\widehat{W}(t)$ ее преобразование Фурье-Стилтьеса, через $\exp \{W\}=\sum_{k=0}^{\infty} W^{k} / k$ ! экспоненциальную меру; $|W|=\sup _{x}|W\{(-\infty, x)\}|$ является аналогом равномерного расстояния, $\|W\|$ означает вариационную норму меры $W$. Вариационная норма (полная вариация) эквивалентна супремуму по всем борелевским множествам. Пусть $\lambda>0, F \in \mathscr{F}$. Тогда $\exp \{\lambda(F-E)\}$ называется обобщенным распределением Пуассона. Для $F \in \mathscr{F}$ и натурального $n$ введем

${ }^{*}$ Вильнюсский университет, кафедра математической статистики, Наугардуко 24 , Вильнюс, Литва. 
множество всех сопровождающих распределений:

$$
\mathscr{D}_{n}^{\alpha}(F)=\left\{E_{n \alpha} \exp \left\{n\left(F E_{-\alpha}-E\right)\right\}, \alpha \in \mathbf{R}\right\} .
$$

Очевидно, что $\mathscr{D}_{n}^{\alpha}(F)$ является подмножеством всех обобщенных распределений Пуассона.

В 1955 г. Ю. В. Прохоров [25] доказал, что для любого $F$ существует последовательность безгранично делимых распределений, близкая к $F^{n}$. А. Н. Колмогоров [15] доказал, что эта аппроксимация равномерна по всему классу $\mathscr{F}$ (первая равномерная теорема Колмогорова). Точность аппроксимации в первой равномерной теореме Колмогорова (общий случай и подклассы $\mathscr{F ) ~ р а с с м а т р и в а л а с ь ~ в ~ р а б о т а х ~ Ю . ~ В . ~ П р о х о р о - ~}$ ва, А. Н. Колмогорова, Л. Д. Мешалкина, Л. Ле Кама, И. А. Ибрагимова и Э. Л. Пресмана, Т. В. Арака, А. Ю. Зайцева и многих других (обзор полученных результатов можно найти в [4]). Проблема была решена Т. В. Араком [2], [3], который доказал, что

$$
C_{1} n^{-2 / 3} \leqslant \sup _{F \in \mathscr{F}} \inf _{D \in \mathrm{D}}\left|F^{n}-D\right| \leqslant C_{2} n^{-2 / 3} .
$$

Однако безгранично делимое распределение в (2) имеет очень сложную структуру. С другой стороны, сопровождающие законы имеют гораздо более простую структуру, хотя в общем случае обеспечивают меньшую точность. Л. Ле Кам [18] (оценка сверху) и И. А. Ибрагимов и Э. Л. Пресман [14] (оценка снизу) установили, что

$$
C_{3} n^{-1 / 3} \leqslant \sup _{F \in \mathscr{F} \alpha} \inf \left|F^{n}-E_{n \alpha} \exp \left\{n\left(F E_{-\alpha}-E\right)\right\}\right| \leqslant C_{4} n^{-1 / 3} .
$$

Отметим, что для некоторых подклассов $\mathscr{F}$ множество $\mathscr{D}_{n}^{\alpha}(F)$ может вполне заменить класс D. Например, Т. В. Арак [1] показал, что

$$
\sup _{F \in \mathscr{F}_{+}}\left|F^{n}-\exp \{n(F-E)\}\right| \leqslant C_{5} n^{-1} .
$$

Сходные проблемы рассматривались в [29], [30]. Многомерный аналог (2) и многомерное обобщение (4) были получены Э. Л. Пресманом [22] и А. Ю. Зайцевым [11].

Аппроксимацию $F^{n}$ можно существенно уточнить для фиксированного $F$, т.е. для $F$, не зависящего от $n$ (схема последовательностей), см. [13], [19], [27]-[29] и приведенную там библиографию. А. Ю. Зайцев [13] поставил вопрос о точности наилучшего приближения в схеме последовательностей. Он выдвинул гипотезу, что

$$
\begin{aligned}
\inf _{D \in D}\left|F^{n}-D\right| & \leqslant C_{6}(F) n^{-1}, \\
\inf _{\alpha}\left|F^{n}-E_{n \alpha} \exp \left\{n\left(F E_{-\alpha}-E\right)\right\}\right| & \leqslant C_{7}(F) n^{-1 / 2} .
\end{aligned}
$$


Известно, что в общем случае оценки (5) и (6) нельзя улучшить. С другой стороны, (5) справедливо для биномиального распределения, см. [23]. Более того, если распределение $F$ имеет достаточно много конечных моментов, то, очевидно, (6) имеет место (достаточно дважды применить центральную предельную теорему).

Как правило, нормальное приближение использует существование по крайней мере конечного второго момента. Для сопровождающих распределений подобное условие гораздо слабее. В настоящее время наилучший результат в этом направлении принадлежит А. Ю. Зайцеву [13]. Сформулируем его. Пусть

$$
\int_{-\infty}^{\infty} x F\{d x\}=0, \quad \int_{-\infty}^{\infty}|x|^{1+\delta} F\{d x\}<\infty
$$

для некоторого $0<\delta \leqslant 1$. Тогда

$$
\left|F^{n}-\exp \{n(F-E)\}\right| \leqslant C_{8}(F) n^{-\varkappa}
$$

где

$$
\varkappa=\left\{\begin{array}{l}
\delta, \text { если } 0<\delta \leqslant \frac{1}{2}, \\
\frac{1}{2}, \text { если } \frac{1}{2} \leqslant \delta \leqslant 1 .
\end{array}\right.
$$

С самого начала стоял вопрос, можно ли заменить равномерное расстояние в (2) (или в (3)) на более сильное расстояние по вариации, см. $[24],[25]$. Исследования в этом направлении в основном велись для решетчатых величин и для величин, удовлетворяющих условию (С), см. [27], [28], [31]. А. Ю. Зайцев [12] доказал, что в общем случае (в схеме серий) невозможен никакой аналог (2) или (3). Он также показал, что то же самое можно сказать о вышеупомянутых подклассах $\mathscr{F}$. Тем не менее, для фиксированного $F$ эта проблема имеет смысл и допускает (по крайней мере частные) решения. Например, из результатов [31] следует, что для $F \in \mathscr{F}_{+}$, сконцентрированного в целых числах и имеющего три конечных момента,

$$
\left\|F^{n}-\exp \{n(F-E)\}\right\| \leqslant C_{9}(F) n^{-1} .
$$

В настоящей заметке мы покажем, что для определенных подклассов $\mathscr{F}$ оценки (5) и (6) имеют место даже для расстояния по вариации (т.е. для всех борелевских множеств). Тем самым гипотеза Зайцева будет частично подтверждена. Полученные результаты можно рассматривать и как количественное уточнение утверждения Прохорова [25] об аппроксимации по вариации. Мы также дадим несколько примеров асимптотических разложений типа Бергстрема, когда разложения строятся с помощью добавления дополнительных членов к основной аппроксимации или в ее экспоненте.

В дальнейшем будем считать, что $F$ не зависит от $n$. 
2. Аппроксимация при выполнении условия (C). Условие Крамера (C) формулируется следующим образом:

$$
\limsup _{|t| \rightarrow \infty}|\widehat{F}(t)|<1
$$

Известно (см. [29]), что при выполнении условия (С) возможно достигнуть точности порядка $n^{-1}$, если в (7) потребовать $\delta=1$. Уточняя этот результат, мы получим оценки для всех $0<\delta \leqslant 1$. В отличие от $(8)$, в оценке не будет излома по отношению к $\delta$.

Теорема 1. Пусть $F$ удовлетворяет условиям (C) $u$ (7). Тогда

$$
\left|F^{n}-\exp \{n(F-E)\}\right| \leqslant C_{10}(F) n^{-\delta} .
$$

Все доказательства содержатся в п. 4.

Рассмотрим теперь оценки по вариации. Будем исследовать множество абсолютно непрерывных законов, имеющих ограниченные плотности. Заметим, что $\exp \{\lambda(F-E)\}=E \exp \{-\lambda\}+\cdots$, и, следовательно, имеется небольшой скачок в нуле. Поэтому неясно может ли сопровождающий закон быть достаточно близким к $F^{n}$ для всех борелевских множеств. Аналогично [28] мы будем для аппроксимаций применять сглаженный сопровождающий закон. Как и выше, будем считать, что существует конечный абсолютный момент только порядка $1+\delta$. Обозначим через $\Phi_{1 n}$ нормальное распределение с характеристической функцией $\exp \left\{-t^{2} /(2 n)\right\}$.

Теорема 2. Пусть $F$ имеет ограниченную плотность, и пусть выполняется условие (7). Тогда

$$
\left\|F^{n}-\Phi_{1 n} \exp \{n(F-E)\}\right\| \leqslant C_{11}(F) n^{-(5 \delta-1) / 4}
$$

Сравнивая (10) и (11), видим, что при всех $0<\delta<1$ для равномерной метрики достигается более высокая точность, нежели для полной вариации.

Если $F \in \mathscr{F}_{+}$, то точность в (11) можно улучшить.

Теорема 3. Пусть $F \in \mathscr{F}_{+}$имеет ограниченную плотность, $u$ пусть выполняется условие (7). Тогда

$$
\left\|F^{n}-\Phi_{1 n} \exp \{n(F-E)\}\right\| \leqslant C_{12}(F) n^{-(3+\delta) / 4} \text {. }
$$

В условиях теорем 2 и 3 можно получить оценки и для плотностей. Пусть $p_{n}(x)$ и $g_{n}(x)$ являются плотностями распределений $F^{n}$ и $\Phi_{1 n} \exp \{n(F-E)\}$.

Теорема 4. Пусть $F$ имеет ограниченную плотность и пусть выполняется условие (7). Тогда

$$
\sup _{x}\left|p_{n}(x)-g_{n}(x)\right| \leqslant C_{13}(F) n^{-\delta-1 / 2} .
$$


Eсли $F \in \mathscr{F}_{+}$имеет ограниченную плотность, то

$$
\sup _{x}\left|p_{n}(x)-g_{n}(x)\right| \leqslant C_{14}(F) n^{-3 / 2} .
$$

Как видно, оценки (4), (14) не требуют существования моментов. Для выполнения (12) нужно предположить существование по крайней мере абсолютного момента порядка $1+\delta$. Неясно, насколько это условие существенно для оценок по вариации. Отметим также, что для $\delta=1$ оценка, аналогичная оценке (13), была получена в [27].

В наиболее классических случаях асимптотические разложения связываются с существованием конечных моментов высших порядков. Однако возможно построить разложения даже при минимальных ограничениях на $F$, см. [5], [10], [20], [30], [31]. В настоящей заметке мы используем короткое разложение, которое аналогично разложению из [10].

Теорема 5. Пусть F удовлетворяет условиям (C) $u$ (7). Тогда

$$
\left|F^{n}-\exp \{n(F-E)\}\left(E-\frac{n(F-E)^{2}}{2}\right)\right| \leqslant C_{15}(F) n^{-2 \delta} .
$$

Отметим, что в (15) ограничения точно такие же, как и в (10). Конечно, если $F$ имеет ограниченную плотность, то теорему 5 можно переформулировать для полной вариации или для плотностей.

Рассмотрим теперь пуассоновские меры со знаком. Под пуассоновской мерой со знаком мы подразумеваем $\exp \{\lambda(F-E)\}$, где $F \in \mathscr{F}$ и $\lambda \in \mathbf{R}$ (т.е. $\lambda$ может быть отрицательным). Пуассоновские аппроксимации со знаком были введены в 1983 г. Э. Л. Пресманом [23] и П. Корния [16] и с тех пор неоднократно успешно применялись (см. [6], [7], [9], [17], $[32],[33])$. Можно рассматривать пуассоновские меры со знаком как специальный тип асимптотических разложений, когда разложения строятся в экспоненте. Однако имеются некоторые сушественные отличия от обычных разложений:

a) пуассоновские меры со знаком всегда безгранично делимы,

б) по сравнению с обычными разложениями они, как правило, обеспечивают более точные оценки,

в) как правило, из них можно вывести другие известные разложения (например, разложение Эджворта),

г) часто пуассоновские аппроксимации со знаком имеют более простое строение.

Мы приведем лишь один пример пуассоновской аппроксимации со знаком.

Теорема 6. Пусть F удовлетворяет условиям (C) и (7). Тогда

$$
\left|F^{n}-\Phi_{1 n} \exp \left\{n(F-E)-\frac{n(F-E)^{2}}{2}\right\}\right| \leqslant C_{16}(F) n^{-(3 \delta+1) / 2} .
$$


Примечательно, что для всех $0<\delta<1$ точность аппроксимации в (16) выше, чем в (15). Если $F$ имеет больше чем три конечных абсолютных момента, то пуассоновская аппроксимация со знаком может быть существенно упрощена, см. [33].

3. Аппроксимация решетчатых распределений. В дальнейшем обозначим через $\mu, \sigma^{2}, \Gamma_{3}$ первые три семиинварианта распределения $F$, т.е.

$$
\ln \widehat{F}(t)=(i t) \mu+\frac{(i t)^{2} \sigma^{2}}{2}+\frac{(i t)^{3} \Gamma_{3}}{6}+\cdots .
$$

Предположим, что $F$ не только не зависит от $n$, но и

$$
\left(\mathrm{L}_{1}\right) \begin{aligned}
& \text { сконцентрировано в целых числах и } \\
& \text { максимальный шаг распределения } F \text { равен } 1 .
\end{aligned}
$$

Очевидно, что распределение $F$ невырождено. Более того, любое решетчатое распределение после подходящего центрирования и нормирования будет удовлетворять условию $\left(L_{1}\right)$. Поэтому все последующие результаты очевидным образом распространяются на другие решетчатые законы.

Аппроксимируя биномиальное распределение классом D, Э. Л. Пресман [23] для расстояния по вариации получил точность аппроксимации порядка $C(F) n^{-1}$. Поэтому можно ожидать, что для решетчатых распределений гипотеза Зайцева (5) должна быть справедлива и для полной вариации. Ниже мы докажем (5) для распределений с четвертым конечным моментом.

Для аппроксимации нам понадобится более сложное распределение, нежели сопровождающий закон. Применим тот подход, который был использован в [2] при решении первой равномерной проблемы Колмогорова.

Из $\left(L_{1}\right)$ следует, что существуют целые числа $a<b$ такие, что $F\{a\} F\{b\}>0$. Пусть $0 \leqslant \beta<1$ такое, что

$$
\gamma=n \mu+\beta \in \mathbf{Z} \text {. }
$$

Определим $G$ как меру конечной вариации с преобразованием ФурьеСтилтьеса:

$$
\begin{gathered}
\widehat{G}(t)=\exp \left\{i t \frac{\gamma}{n}-\frac{\beta}{n(b-a)}\left(e^{\imath t(b-a)}-1\right)+\frac{\beta}{6 n(b-a)}\left(e^{2 \imath t(b-a)}-1\right)\right. \\
\left.+\frac{\beta}{3 n(b-a)}\left(e^{-\imath t(b-a)}-1\right)+\frac{1}{6}\left(\widehat{F}(2 t) \widehat{F}^{2}(-t)-1\right)\right\} .
\end{gathered}
$$

Теорема 7. Пусть $F$ имеет четвертый конечный момент и пусть выполняется условие $\left(L_{1}\right)$. Тогда для $n \geqslant 6(F\{b\})^{-2}(F\{a\})^{-1}$ имеем

$$
\left\|F^{n}-G^{n}\right\| \leqslant C_{17}(F) n^{-1} .
$$


Ограничение на $n$ наложено исключительно для того, чтобы обеспечить $G \in \mathscr{F}$. В самом деле, $\widehat{F}(2 t) \widehat{F}^{2}(-t)$ соответствует распределению, при котором точке $b-a$ соответствует вероятность $\widetilde{p} \geqslant(F\{b\})^{2} F\{a\}$. Но тогда из условия $n \geqslant 6(F\{b\})^{-2}(F\{a\})^{-1}$ следует $n \tilde{p} / 6 \geqslant \beta /(b-a)$ и, тем самым, $G \in \mathscr{F}$.

Неясно, имеет ли место аналог (6) для расстояния полной вариации при аппроксимации решетчатых распределений. До сих пор аналог (6) был установлен лишь для решетчатых распределений с нулевым средним, см. [28]. Отметим, что далеко не всякое решетчатое распределение можно центрировать так, чтобы и среднее превратилось в ноль, и решетчатость сохранилась. Однако, несколько расширив класс $\mathscr{D}_{n}^{\alpha}(F)$, можно получить некоторый аналог (6).

Теорема 8. Пусть $F$ имеет конечный третий абсолютный момент, и пусть выполняется условие $\left(L_{1}\right)$. Тогда

$$
\left\|F^{n}-E_{\nu} \exp \{n u(F-E)\}\right\| \leqslant C_{18}(F) n^{-1 / 2} .
$$

Здесь $\nu=[n(1-u) \mu], u=\sigma^{2} / A_{2}, A_{2}=\int x^{2} d F, a[\cdot]$ означает челую часть числа.

Конечно, пуассоновские аппроксимации со знаком применимы и для решетчатых распределений. Покажем, как в этом случае можно упростить распределение $G$. В результате получим меру со знаком, но без $\widehat{F}(2 t) \widehat{F}^{2}(-t)$ в экспоненте. Пусть $0 \leqslant \widetilde{\beta}<1$ такое, что $\widetilde{\gamma}=n \mu-n \Gamma_{3}+\widetilde{\beta} \in \mathbf{Z}$. Положим

$$
\begin{aligned}
\widehat{G}_{1}(t)=\exp & \left\{i t \frac{\widetilde{\gamma}}{n}+\left(\frac{\sigma^{2}+\Gamma_{3}}{2}-\frac{\widetilde{\beta}}{n}\right)\left(e^{i t}-1\right)\right. \\
& \left.+\left(\frac{\sigma^{2}-\Gamma_{3}}{2}+\frac{\widetilde{\beta}}{3 n}\right)\left(e^{-i t}-1\right)+\frac{\widetilde{\beta}}{6 n}\left(e^{2 i t}-1\right)\right\} .
\end{aligned}
$$

Очевидно, что $G_{1}$ является сверткой трех мер пуассоновского типа.

Теорема 9. Пусть $F$ имеет конечный четвертый момент, и пусть выполняется условие $\left(L_{1}\right)$. Тогда

$$
\left\|F^{n}-G_{1}^{n}\right\| \leqslant C_{19}(F) n^{-1} .
$$

Конечно, можно построить асимптотические разложения и $\kappa$ обобщенной мере Пуассона, не только в ее экспоненте, см. [17].

В условиях предыдущих теорем можно получить и локальные оценки. Покажем это на некоторых аппроксимациях.

Теорема 10. Пусть $F$ имеет конечный четвертый момент, и пусть выполняется условие $\left(L_{1}\right)$. Тогда

$$
\begin{gathered}
\sup _{m}\left|F^{n}\{m\}-G^{n}\{m\}\right| \leqslant C_{20}(F) n^{-3 / 2}, \\
\sup _{m}\left|F^{n}\{m\}-G_{1}^{n}\{m\}\right| \leqslant C_{21}(F) n^{-3 / 2} .
\end{gathered}
$$


Конечно, $G$ может, при малых $n$, оказаться мерой со знаком, но при $n \geqslant 6(F\{b\})^{-2}(F\{a\})^{-1}$ будет $G \in \mathscr{F}$.

4. Доказательства. Д о к а з а те л ь с т в о теорем $1,4,5$ и 6. В дальнейшем для краткости будем опускать индексы для $C(F)$ (т.е. символ $C(F)$ будем использовать для различных постоянных) и зависимость от $t$ для преобразований Фурье-Стилтьеса. Без потери общности предположим, что $n \geqslant 4$. Из условия (С) следует, что для любого $\varepsilon=\varepsilon(F)>0$ и всех $|t|>\varepsilon$

$$
\begin{aligned}
& |\widehat{F}| \leqslant e^{-C(F)}, \quad|\exp \{\widehat{F}-1\}| \leqslant \exp \{|\widehat{F}|-1\} \leqslant e^{-C(F)}, \\
& \left|\exp \left\{(\widehat{F}-1)-\frac{(\widehat{F}-1)^{2}}{2}\right\}\right| \leqslant \exp \left\{\frac{|\widehat{F}|^{2}-1}{2}\right\} \leqslant e^{-C(F)} .
\end{aligned}
$$

С другой стороны, $F-$ невырожденное распределение. Следовательно, для малого $\varepsilon=\varepsilon(F)$ и $|t| \leqslant \varepsilon$,

$$
\max \left(|\widehat{F}|,|\exp \{\widehat{F}-1\}|, \exp \left\{\frac{|\widehat{F}|^{2}-1}{2}\right\}\right) \leqslant \exp \left\{-C(F) t^{2}\right\} .
$$

Пусть $W$ - некоторая мера ограниченной вариации. Тогда, по лемме сглаживания Эссеена (см. [21, с. 149]), для любого $T>0$

$$
\begin{aligned}
\left|F^{n}-W\right| \leqslant & C \int_{0}^{\varepsilon} \frac{|\widehat{F}-\widehat{W}|}{|t|} d t+C \int_{\varepsilon}^{T} \frac{|\widehat{F}-\widehat{W}|}{|t|} d t \\
& +C T \sup _{x} \int_{|y| \leqslant C / T}|W\{[x, x+y)\}| d y .
\end{aligned}
$$

Положим $T=n^{4}$, и пусть

$$
\begin{aligned}
& U_{1}=\exp \{F-E\}, \quad U_{2}=U_{1}^{n}\left(E-\frac{n(F-E)^{2}}{2}\right), \\
& U_{3}^{n}=\Phi_{1 n} \exp \left\{n(F-E)-\frac{n(F-E)^{2}}{2}\right\} .
\end{aligned}
$$

Из (26)-(29) и свойств функции концентрации получаем

$$
\begin{aligned}
\left|F^{n}-U_{1}^{n}\right| \leqslant & C(F) \int_{0}^{\varepsilon} \frac{\left|\widehat{F}^{n}-\widehat{U}_{1}^{n}\right|}{t} d t+C(F) n^{-2} \\
& +C(F) Q\left(U_{1}^{n}, T^{-1}\right), \\
\left|F^{n}-U_{2}\right| \leqslant & C(F) \int_{0}^{\varepsilon} \frac{\left|\widehat{F}^{n}-\widehat{U}_{2}\right|}{t} d t+C(F) n^{-2} \\
& +C(F) \mid E-\frac{n(F-E)^{2}}{2} \| Q\left(U_{1}^{n}, T^{-1}\right), \\
\left|F^{n}-U_{3}^{n}\right| \leqslant & C(F) \int_{0}^{\varepsilon} \frac{\left|\widehat{F}^{n}-\widehat{U}_{3}^{n}\right|}{t} d t+C(F) n^{-2} \\
& +\frac{C(F)}{T} \int_{-\infty}^{\infty} e^{-t^{2} / 2 n} d t .
\end{aligned}
$$


Из свойств функции концентрации (см. [4, гл. 2]) и из (26), (28) следует

$$
\begin{aligned}
Q\left(U_{1}^{n}, T^{-1}\right) & \leqslant \frac{C}{T} \int_{-T}^{T}\left|\widehat{U}_{1}^{n}\right| d t \leqslant \frac{C}{T}\left(\int_{0}^{\varepsilon}+\int_{\varepsilon}^{T}\right) \\
& \leqslant \frac{C}{T} \int_{0}^{\infty} e^{-C(F) t^{2} n} d t+C(F) T e^{-n C(F)} \leqslant C(F) n^{-2} .
\end{aligned}
$$

Из (31)-(34) видно, что достаточно получить требуемые оценки только в окрестности нуля. Но в [13] показано, что из условия (7) следует, что для всех $t$

$$
|\widehat{F}(t)-1| \leqslant C(F)|t|^{1+\delta} .
$$

Таким образом, для $|t| \leqslant \varepsilon$

$$
\begin{aligned}
\left|\widehat{F}^{n}-\widehat{U}_{1}^{n}\right| & \leqslant n e^{-(n-1) C(F) t^{2}}\left|\widehat{F}-\widehat{U}_{1}\right| \leqslant C(F) n e^{-C(F) n t^{2}}|\widehat{F}-1|^{2} \\
& \leqslant C(F) e^{-C(F) n t^{2}} n|t|^{2+2 \delta}
\end{aligned}
$$

Аналогично,

$$
\left|\widehat{F}^{n}-\widehat{U}_{3}^{n}\right| \leqslant C(F) e^{-C(F) n t^{2}} n\left(|t|^{3+3 \delta}+t^{2} n^{-2}\right) .
$$

Учитывая тождество Бергстрема [5]

$$
A^{n}=\sum_{j=0}^{s}\left(\begin{array}{l}
n \\
j
\end{array}\right) B^{n-j}(A-B)^{j}+\sum_{j=s+1}^{n}\left(\begin{array}{c}
j-1 \\
s
\end{array}\right) A^{n-j} B^{j-s-1}(A-B)^{s+1}
$$

и экспоненциальную структуру распределения $U_{1}$, для $|t| \leqslant \varepsilon$ получаем

$$
\begin{aligned}
\left|\widehat{F}^{n}-\widehat{U}_{2}\right| \leqslant & \left|\widehat{F}^{n}-\widehat{U}_{1}^{n}-n \widehat{U}_{1}^{n-1}\left(\widehat{F}-\widehat{U}_{1}\right)\right|+n\left|\left(\widehat{U}_{1}^{n-1}-\widehat{U}_{1}^{n}\right)\left(\widehat{F}-\widehat{U}_{1}\right)\right| \\
& +n\left|\widehat{U}_{1}^{n}\left(\widehat{F}-\widehat{U}_{1}+\frac{(\widehat{F}-1)^{2}}{2}\right)\right| \\
\leqslant & C(F) \exp \left\{-C(F) n t^{2}\right\}\left(n^{2}|\widehat{F}-1|^{4}+n|\widehat{F}-1|^{3}\right) \\
\leqslant & C(F) \exp \left\{-C(F) n t^{2}\right\}|t|^{4 \delta} .
\end{aligned}
$$

Из (36)-(38) и (31)-(33) нетрудно вывести утверждения теорем 1,5 и 6.

Если $F$ имеет ограниченную плотность, то

$$
\int_{-\infty}^{\infty}|\widehat{F}(t)|^{2} d t \leqslant C(F)
$$

Таким образом, по формуле обрашения и с учетом (28)

$$
\begin{aligned}
& \sup _{x}\left|p_{n}(x)-g_{n}(x)\right| \leqslant \int_{-\infty}^{\infty}\left|\widehat{F}^{n}-e^{-t^{2} / 2 n} \widehat{U}_{1}^{n}\right| d t \\
& \leqslant \int_{|t| \leqslant \varepsilon}\left|\widehat{F}^{n}-e^{-t^{2} / 2 n} \widehat{U}_{1}^{n}\right| d t \\
& \quad+\int_{|t|>\varepsilon}|\widehat{F}|^{n-2}|\widehat{F}|^{2} d t+2 \int_{\varepsilon}^{T}\left|\widehat{U}_{1}\right|^{n} d t+2 \int_{T}^{\infty} e^{-t^{2} / 2 n} d t \\
& \leqslant C(F) \int_{0}^{\varepsilon}\left|\widehat{F}-\widehat{U}_{1}^{n}\right| d t+C(F) n^{-2}
\end{aligned}
$$


Из (40) и (36) следует (13). Если $F \in \mathscr{F}_{+}$, то для $|t| \leqslant \varepsilon$

$$
\left|\widehat{F}^{n}-\widehat{U}_{1}^{n}\right| \leqslant C n \exp \{(n-1)(\widehat{F}-1)\}(1-\widehat{F})^{2} \leqslant C n^{-1} \exp \left\{-C(F) n t^{2}\right\} .
$$

Из (41) и (36) следует (14). Теорема 4 доказана.

Д о к а 3 а т е л ь с т в о теорем 2 и 3 . Нам понадобится следуюшее вспомогательное утверждение из $[4$, с. 136$]$.

Лемма 1. Пусть $H_{1}, H_{2} \in \mathscr{F}$ имеют плотности $h_{1}(x)$ u $h_{2}(x)$. ПІусть $\int_{\mathbf{R}}|x|\left|h_{1}(x)-h_{2}(x)\right| d x<\infty$. Тогдa

$$
\left\|H_{1}-H_{2}\right\| \leqslant \frac{1}{2}\left(\int_{-\infty}^{\infty}\left|\widehat{H}_{1}(t)-\widehat{H}_{2}(t)\right|^{2} d t \int_{-\infty}^{\infty}\left|\left(\widehat{H}_{1}(t)-\widehat{H}_{2}(t)\right)^{\prime}\right|^{2} d t\right)^{1 / 4} .
$$

Точно так же, как и при доказательстве $(36)$, для $|t| \leqslant \varepsilon$ получаем

$$
\left|\widehat{F}^{n}-e^{-t^{2} / 2 n} \widehat{U}_{1}^{n}\right| \leqslant C(F) e^{-C(F) n t^{2}}\left(n|t|^{2+2 \delta}+\frac{t^{2}}{n}\right) .
$$

Аналогично доказательству (35) (см. [13]) имеем

$$
\begin{aligned}
\left|(\widehat{F}(t))^{\prime}\right| & =\left|i \int_{-\infty}^{\infty} x e^{i t x} F\{d x\}\right|=\left|i \int_{-\infty}^{\infty} x\left(e^{i t x}-1\right) F\{d x\}\right| \\
& \leqslant\left|\int_{|x t| \leqslant 1}\right|+\left|\int_{|x t|>1}\right| \\
& \leqslant|t| \int_{|x t| \leqslant 1} x^{2} F\{d x\}+2 \int_{|x t|>1}|x| F\{d x\} \\
& \leqslant 3|t|^{\delta} \int_{-\infty}^{\infty}|x|^{1+\delta} F\{d x\}=C(F)|t|^{\delta} .
\end{aligned}
$$

Поэтому для $|t| \leqslant \varepsilon$

$$
\left|\left(\widehat{F}^{n}-e^{-t^{2} / 2 n} \widehat{U}_{1}^{n}\right)^{\prime}\right| \leqslant C(F) e^{-C(F) n t^{2}}\left(n^{2}|t|^{2+3 \delta}+n|t|^{1+2 \delta}+|t|^{2+\delta}+\frac{|t|}{n}\right) .
$$

Из (26), (29) и (43) следует, что для доказательства теорем 2 и 3 достаточно получить требуемые оценки лишь при $|t| \leqslant \varepsilon$. Но тогда (11) следует из (43) и (44). В случае $F \in \mathscr{F}_{+}$нужно использовать оценки

$$
\begin{aligned}
\left|\widehat{F}^{n}-e^{-t^{2} / 2 n} \widehat{U}_{1}^{n}\right| & \leqslant C(F) e^{-C(F) n t^{2}} n^{-1}, \\
\left|\left(\widehat{F}^{n}-e^{-t^{2} / 2 n} \widehat{U}_{1}^{n}\right)^{\prime}\right| & \leqslant C(F) e^{-C(F) n t^{2}}\left(|t|^{\delta}+\frac{|t|}{n}\right) .
\end{aligned}
$$

Д о к а з а т е ль с т в о теорем 7-10. Для любой меры $\Delta$, сконцентрированной в целых числах, будет $\|\Delta\|=\sum_{k}|\Delta\{k\}|$. Для оценок по вариации используем следуюшее вспомогательное утверждение.

Лемма 2. Пусть $\widehat{\Delta}(t)=\sum_{j} \Delta\{j\} \exp \{i t j\}, \sum_{j}|\Delta\{j\}|<\infty$. Тогда $\partial л g s>0, v \in \mathbf{R}$

$$
\left(\sum_{j=-\infty}^{\infty}|\Delta\{j\}|\right)^{2} \leqslant\left(1+\frac{1}{s}\right) C \int_{-\pi}^{\pi}\left(s|\widehat{\Delta}(t)|^{2}+\frac{1}{s}\left|\left(\widehat{\Delta}(t) e^{-i t v}\right)^{\prime}\right|^{2}\right) d t .
$$


Доказательство леммы 2 можно найти в [24].

Нетрудно проверить, что для $|t| \leqslant \varepsilon=\varepsilon(F)$, где $\varepsilon$ достаточно мало:

$$
\begin{aligned}
& \left|\widehat{F}^{n}(t)\right| \leqslant e^{-C(F) n t^{2}}, \quad\left|\widehat{G}^{n}(t)\right| \leqslant C(F) e^{-C(F) n t^{2}}, \\
& |\exp \{i t \nu+n u(\widehat{F}(t)-1)\}| \leqslant e^{-C(F) n t^{2}} .
\end{aligned}
$$

Более того, для всех $|t| \leqslant \pi$

$$
\left|\widehat{G}_{1}^{n}(t)\right| \leqslant C(F) \exp \left\{-2 n \sigma^{2} \sin ^{2}\left(\frac{t}{2}\right)\right\} \leqslant C(F) e^{-C(F) n t^{2}} .
$$

В силу условия $\left(L_{1}\right)$ (см., например, $[8$, с. 66]) для $\varepsilon<|t| \leqslant \pi$,

$$
\begin{aligned}
& \left|\widehat{F}^{n}(t)\right| \leqslant e^{-C(F) n}, \quad\left|\widehat{G}^{n}(t)\right| \leqslant C(F) e^{-C(F) n} \\
& \exp \{i t \nu+n u(\widehat{F}(t)-1)\} \mid \leqslant e^{-C(F) n}
\end{aligned}
$$

Из леммы 2 неравенств (49) и (50) видим, что достаточно получить требуемые оценки только для $|t| \leqslant \varepsilon$. Но простое разложение $\widehat{F}(t)$ и $\widehat{G}(t)$ по степеням $t$ дает

$$
\begin{aligned}
\left|\widehat{F}^{n}-\widehat{G}^{n}\right| & \leqslant C(F) e^{-C(F) n t^{2}} n|\widehat{F}-\widehat{G}| \\
& \leqslant C(F) e^{-C(F) n t^{2} n t^{4}} \\
& \leqslant C(F) e^{-C(F) n t^{2}} n^{-1} \\
\left|\left(\left(\widehat{F} e^{-i t \mu}\right)^{n}-\left(\widehat{G} e^{-i t \mu}\right)^{n}\right)^{\prime}\right| & \leqslant C(F) n e^{-C(F) n t^{2}}|t|^{3} \\
& \leqslant C(F) e^{-C(F) n t^{2}} n^{-1 / 2}, \\
\left|\widehat{F}^{n}-\widehat{G}_{1}^{n}\right| & \leqslant C(F) e^{-C(F) n t^{2}} n^{-1} \\
\left|\left(\left(\widehat{F} e^{-i t \mu}\right)^{n}-\left(\widehat{G}_{1} e^{-i t \mu}\right)^{n}\right)^{\prime}\right| & \leqslant C(F) e^{-C(F) n t^{2}} n^{-1 / 2} .
\end{aligned}
$$

Из (51)-(53) и леммы 2 (с $s=\sqrt{n}$ и $v=n \mu$ ) получаем (20) и (22). Теорема 10 доказывается прямым применением формулы обращения для вероятностей и оценок (51), (53).

Для доказательства теоремы 8 отметим, что

$$
\frac{i t \nu}{n}+u(\hat{F}(t)-1)=i t \mu-\frac{\sigma^{2} t^{2}}{2}+\theta_{1}|t|^{3}+\frac{\theta_{2}|t|}{n} .
$$

Здесь $\left|\theta_{1,2}\right| \leqslant C(F)$. Таким образом, для $|t| \leqslant \varepsilon$

$$
\begin{aligned}
& \left|\widehat{F}^{n}-\exp \{i t \nu+n u(\widehat{F}-1)\}\right| \\
& \quad \leqslant n C(F) e^{-C(F) n t^{2}}\left(\left|\widehat{F}-\exp \left\{i t \mu-\frac{\sigma^{2} t^{2}}{2}\right\}\right|+C(F)|t|^{3}+C(F) \frac{|t|}{n}\right) \\
& \quad \leqslant C(F) e^{-C(F) n t^{2}} n^{-1 / 2} .
\end{aligned}
$$


Аналогично,

$$
\left|\left(\left(\widehat{F} e^{-i t \mu}\right)^{n}-\exp \{i t(\nu-n \mu)+n u(\widehat{F}-1)\}\right)^{\prime}\right| \leqslant C(F) e^{-C(F) n t^{2}}
$$

Для завершения доказательства (23) достаточно применить лемму 2 с $s=\sqrt{n}$ и $v=n \mu$.

\section{СПИСОК ЛИТЕРАТУРЫ}

1. Арак Т.В. О сближении $n$-кратных сверток распределений, имеющих неотрицательную характеристическую функцию, с сопровождающими законами. Теория вероятн. и ее примен., 1980, т. XXV, в. 2, с. 225-246.

2. Арак Т.В. О скорости сходимости в равномерной предельной теореме Колмогорова I; II. - Теория вероятн. и ее примен., 1981, т. XXVI, в. 2, с. 225-245; в. 3, c. $449-463$.

3. Арак T.В. Уточнение нижней оценки для скорости сходимости в равномерной предельной теореме Колмогорова. - Теория вероятн. и ее примен., 1982, т. XXVII, в. 4, с. 767-772.

4. Арак T.В., Зайцев А. Ю. Равномерные предельные теоремы для сумм независимых случайных величин. - Тр. Матем. ин-та им. В. А. Стеклова АН СССР, 1986 , т. 174 , с. $1-216$.

5. Bergström $H$. On asymptotic expansion of probability functions. - Skand. Aktuarietidskrift, 1951, v. 1, p. 1-34.

6. Боровков K.A. K вопросу об уточнении пуассоновской аппроксимации. - Теория вероятн. и ее примен., 1988, т. XXXIII, в. 2, с. 364-368.

7. Borovkov K.A., Pfeifer D. Pseudo-Poisson approximation for Markov chains. Stochastic Process. Appl., 1996, v. 61, № 1, p. 163-180.

8. Гнеденко Б. В., Колмогоров А.Н. Предельные распределения для сумм независимых случайных величин. М.-Л.: ГИТТЛ, 1949. 264 с.

9. Hipp C. Improved approximations for the aggregate claims distribution in the individual model. - ASTIN Bull., 1986, v. 16, p. 89-100.

10. Deheuvels $P$., Pfeifer D. A semigroup approach to Poisson approximation. - Ann. Probab., 1986, v. 14, p. 663-676.

11. Зайчев $A . Ю$. Об аппроксимации сверток многомерных симметричных распределений сопровождающими законами. - Записки научн. семинаров ЛОМИ, 1989, т. 177 , с. $55-72$.

12. Зайчев А.Ю. Пример распределения, множество $n$-кратных сверток которого равномерно отделено от множества безгранично делимых законов в смысле расстояния по вариации. - Теория вероятн. и ее примен., 1991, т. 36 , в. 2, с. 356361.

13. Зайчев А.Ю. Аппроксимация сверток сопровождающими законами при существовании моментов невысоких порядков. - Записки научн. семинаров ПОМИ, 1996, т. 228, c. $135-141$.

14. Ибрагимов И.А., Пресман Э.Л. О скорости сближения распределений сумм независимых случайных величин с сопровождающими законами. - Теория вероятн. и ее примен., 1973, т. XVIII, в. 4, с. 753-766.

15. Колмогоров $A$. Н. Две равномерные предельные теоремы для сумм независимых слагаемых. - Теория вероятн. и ее примен., 1956, т. I, в. 4, с. 426-436.

16. Kornya $P$. Distribution of aggregate claims in the Individual Risk Theory model. Society of Actuaries: Transactions, 1983, v. 35, p. 823-858.

17. Круопис Ю. Точность аппроксимации обобщенного биномиального распределения свертками пуассоновских мер. - Литов. матем. сб., 1986, т. 26, в. 1, с. 53-69. 
18. Le Cam $L$. On the distribution of sums of independent random variables. - In: Bernoulli, Bayes, Laplace (Anniversary volume). Berlin: Springer-Verlag, 1965, p. 179-202.

19. Маневич Д. В. Об оценке приближения распределений сумм сопровождающими законами. - Сб. трудов Ташкент. пед. гос. ин-та, 1977, в. 183, с. 37-44.

20. Осипов Л.В. Об асимптотических разложениях для функций распределения сумм независимых случайных величин. - Теория вероятн. и ее примен., 1971, т. XVI, в. 2, с. $328-338$.

21. Петров В.В. Предельные теоремы для сумм независимых случайных величин. M.: Наука, 1987. 320 c.

22. Пресман Э. Л. О многомерном варианте равномерной предельной теоремы Колмогорова. - Теория вероятн. и ее примен., 1973, т. XVIII, в. 2, с. 396-402.

23. Пресман Э.Л. О сближении биномиальных и безгранично делимых распределений. - Теория вероятн. и ее примен., 1983, т. XXVIII, в. 2, с. 372-382.

24. Пресман Э.Л. О сближении по вариации распределения суммы независимых бернуллиевских величин с пуассоновским законом. - Теория вероятн. и ее примен., 1985, т. XXX, в. 2, с. 391-396.

25. Прохоров Ю.В. О суммах одинаково распределенных величин. - ДАН СССР, 1955 , т. 105 , № 4, с. $645-647$.

26. Прохоров Ю. В. О равномерной предельной теореме А. Н. Колмогорова. - Теория вероятн. и ее примен., 1960, т. V, в. 1, с. 103-113.

27. Салахутдинов $P$. О локальных теоремах в приближении распределений сумм безгранично делимыми законами. - Учен. записки Ташкент. пед. ин-та, 1975, B. 141, c. $64-78$.

28. Салахутдинов $P$. К вопросу о сходимости по вариащии. - Учен. записки Ташкент. пед. ин-та, 1975 , в. 141, с. 79-82.

29. Студнев Ю. П. К вопросу о приближении распределений сумм неограниченно делимыми законами. - Теория вероятн. и ее примен., 1960, т. V, в. 4, с. 465-469.

30. Чяканавичюс $B$. Аппроксимация сопровождающими распределениями и асимптотическими разложениями I. - Литов. матем. сб., 1989, т. 29, в. 1, с. 171-178.

31. Čkanavičius $V$. On approximation of integer-valued measures by generalized Poisson measures. - In: Proceedings of the 5th Vilnius Conference on Probability Theory and Mathematical Statistics, v. 1. Vilnius/Utrecht: Mokslas/VSP, 1990, p. 228-237.

32. Cekanavičius V. On multivariate Le Cam theorem and compound Poisson measures. - Statist. Probab. Lett., 1996, v. 25, p. 145-151.

33. Cekanavičius $V$. Asymptotic expansions in the exponent: a compound Poisson approach. - Adv. Appl. Probab. 1997, v. 25, p. 374-387.

Поступила в редакцию 12.V.1997 